\title{
Research on Automatic Communication Technology of Distribution Network
}

\author{
Ke Yuan ${ }^{1, a}$, Zengguang Wei, ${ }^{2, b}$, Xiaowei Shao ${ }^{3, c}$ \\ ${ }^{1}$ Nanchang institute of science \&technology, Nanchang, Jiangxi, 330000 \\ ${ }^{2}$ Nanchang industrial technology research institute, Nanchang, Jiangxi, 330000 \\ ${ }^{3}$ Nanchang institute of science \&technology, Nanchang, Jiangxi, 330000 \\ ${ }^{\mathrm{a}}$ email, ${ }^{\mathrm{b}}$ email, ${ }^{\mathrm{c}}$ email
}

Keywords: Distribution Network, Automatic Communication Technology, Information Science

\begin{abstract}
Distribution automation can choose a variety of communication technologies: fiber optic communications, power line carrier communications, wireless communications, and so on. Because of the characteristics and requirements of automatic communication of distribution network, it is decided that a single communication technology can not be adopted in the communication system, and the choice of different communication technologies and program design have great influence on the real-time, reliability and effectiveness of the communication system, Its construction and operation and maintenance costs are also very different.
\end{abstract}

\section{Introduction}

Distribution automation is the use of modern electronic technology, communication technology, computer technology and network technology, the distribution network data, user data, power grid structure and geographic graphics information integration, constitute a complete automation system to achieve the normal operation of the distribution system and accidents Conditional monitoring, protection, control. The rise of distribution automation in China is mainly due to the long-term urban power grid in the construction of the distribution network is not taken seriously, with a weak network structure, resulting in power generation capacity at the same time for the low capacity of distribution. So the state proposed to actively and steadily promote the distribution network automation. The goal of distribution automation can be attributed to improve the reliability of power supply and improve the quality of power, to ensure the provision of high-quality electricity to users; to improve the overall power supply capacity of urban and rural power grid; to achieve distribution network management automation, a number of management processes to provide information support, Improve the management level and labor productivity; reduce the operation and maintenance costs and various losses, to achieve the economic operation of the distribution network; improve the quality of service for the reform of the electricity market lay a good technical foundation.

\section{Distribution Network Automation Communication System}

The distribution automation system consists of DAS master station, trunk communication network, distribution station, branch communication network and monitoring terminal. The distribution system of distributed automation has the characteristics of monitoring points and scattered. The communication system generally adopts the structure of the trunk channel network and the branch communication network. It is divided into users, line FTU, substation, distribution station, distribution main station and so on. Users (such as power monitoring, automatic reading table) terminal data from the line FTU, distribution station forward, line FTU data forwarded by the distribution station, distribution station directly with the distribution of the main station communication. The automation of the distribution network can be summarized as several items, namely, distribution network data acquisition and monitoring (SCADA), automatic fault isolation and recovery of power supply, voltage and reactive power management, load management, 
automatic meter reading, equipment management, maintenance management, Power outage management, planning and design management, electricity management. The role of the implementation of distribution network automation mainly in the following aspects:

(1) To improve power supply reliability. The use of feeder automation system fault isolation and automatic recovery of power supply function, can reduce the power outage range; Secondly, by improving the distribution network construction, maintenance and accident repair work efficiency, reduce planning and fault power outage time; again, through the grid real-time Monitoring, can be found in time hidden trouble, the implementation of state maintenance, improve equipment reliability, to avoid the occurrence of power failure.

(2) To improve the voltage quality. Power distribution automation system can be a variety of field terminals on the supply voltage changes in real-time monitoring, in order to adjust the operation mode, adjust the transformer tap or switch reactive power compensation capacitor group, to ensure that the user voltage within the qualified range.

(3) To improve the quality of customer service. Power distribution automation system can quickly handle the user's electricity applications, to speed up the user to pay electricity and business inquiries processing speed, to determine the location of power failure failure, causes and power outages, as soon as possible to restore power supply to provide protection.

(4) Improve management efficiency. The automation of distribution can carry out remote real-time monitoring and operation control of the operation status of distribution network equipment, and realize the automation and informationization of distribution production and electricity management, thus improving the working efficiency and quality.

(5) Postpone investment in capital construction. The use of distribution automation technology can effectively adjust the peak and valley load, improve equipment utilization, compression reserve capacity, reduce or delay the capital construction investment.

\section{Distribution Network Automation Communication Technology}

Optical fiber communication is based on the light as the carrier, the optical fiber as the transmission medium communication technology. Optical fiber communication and other communications have the following advantages: transmission bandwidth, communication capacity, transmission loss, anti-electromagnetic interference, networking and flexible. At present, the access of optical fiber communication basically rely on active optical network (Active OpticalNetwork, AON) and passive optical network (Passive Optical Network, PON) two technologies. PON technology due to the passive node, laying and running maintenance costs low, transparent and easy to upgrade business and other advantages of concern.

In recent years, power line communication (PLC) technology has become a new research hotspot in communication system. However, power carrier communication due to time-varying, frequency selectivity and other inherent characteristics, so that in the specific application there are still many problems waiting to be resolved. Power line carrier communication (PLC) refers to the use of special modem to signal the modulation, and then load the signal into the existing power lines for communication technology. As early as the 1920s, power carrier communication began to apply to $10 \mathrm{kV}$ distribution network line communication, the use of power carriers and shock absorbers, in the high-voltage distribution network transmission of voice, control instructions and system status and other information, And formed the relevant international and national standards. For low-voltage distribution networks, many emerging digital technologies, such as spread spectrum communication technology, digital signal processing technology and computer control technology, greatly improve the low-voltage distribution network power carrier communication availability and reliability, so that power carrier communication Technology has a more attractive application prospects.

GPRS (General Packet Radio Service) is a standard technology of packet-switched data. It is a wireless packet switching technology based on GSM system. With GSM network, the transmission speed can reach $115 \mathrm{kbit} / \mathrm{s}$, which is between the second generation And the third generation of a technology, commonly known as 2.5G. GPRS provides end-to-end, wide area wireless IP 
connection, based on data transmission to charge, rather than simply to online time billing. Which is suitable for the frequent transmission of small data and non-frequent transmission of large amounts of data applications, at present, the scope of application in China's power distribution network for the wireless meter reading system, reactive power compensation system, load control systems, such as urban and rural areas, suburban line equipment, And the operation time is not high and do not need to instantaneous action with the terminal.

Global Wireless Access Interoperability (WiMAX) is a wireless broadband metropolitan area network (WMAN) access technology, the physical layer and MAC layer are based on IEEE 802.16 work group developed wireless metropolitan area network technology, to achieve fixed and mobile users High-speed wireless access. The WiMAX network system consists of a core network and an access network. The core network includes network management system, router, AAA proxy server, user database and gateway equipment, mainly to achieve user authentication, roaming, network management and other functions, and provide the interface between other networks. The access network includes a base station (BS), a subscriber station (SS), and a mobile subscriber station (MSS), which are responsible for providing wireless access for WiMAX users [40].

\section{Distribution Network Automation Communication Program}

Distribution communication is divided into three levels: the master station, sub-station and access layer. Master station layer communication mostly uses TCP / IP technology based on the local area network, sub-station communication through the already built SDH / MSTP optical fiber transmission ring network, access layer using EPON as optical access network. The optical line terminal OLT is placed on the distribution station, and each distribution station is located on the SDH / MSTP transmission ring. The distribution station communicates the distribution terminal of the distribution terminal and communicates with the master station through the SDH / MSTP ring. OLT can provide two PON port composed of two separate backup chain, dual PON port can provide high reliability, each open and close, ring network cabinet or column switch on the optical network unit ONU can be connected through the dual PON port To the two chains, each chain of the beam splitter are used $1 \times 2$ non-bisected splitter.

In order to further enhance the reliability and security, important sites can also take two OLT out of a PON port composed of hand-held two chains of the network, in hand-hand protection mode, the failure time can be up to $50 \mathrm{~ms}$ or less The Each ONU through the dual PON port were connected to the two chains, so that you can achieve the backbone of optical fiber, OLT equipment, PON port, optical splitter, branch cable protection of the whole network, the network of any one OLT, any one PON Mouth, any one of the splitter, any one cable failure will not affect the normal use of ONU.

Communication media analysis. At present, the power companies have built the dispatching communication network with SDH / MSTP fiber optic transmission network. The communication between $35 \mathrm{kV}$ and $110 \mathrm{KV}$ substation in the power grid is basically covered by the optical fiber. Therefore, the optical fiber communication network is equipped with $110 \mathrm{kV}$ and $35 \mathrm{kV}$ The following distribution of communication lines extends the foundation. Power system laying fiber can be erected along the distribution line placed under the power conductor, can also be placed in the high-voltage distribution line or the use of power lines, buried cable. But no matter what kind of laying or use what kind of fiber optic cable, as long as the single-mode fiber media, EPON can support. The POS (splitter) in the EPON system is a passive device, so there is little requirement for the placement, which can be buried or buried. It is noteworthy that, in order to save the cost of laying the cable can use the existing distribution network of pipeline resources for overhead lines can be laid with the tower; for the buried line can be laid with the route. The OLT equipment in the chained network is arranged in the distribution station, and a number of POS (1: 2 non-equalizer splitter) is cascaded through a PON port of the OLT, and the "hand- It is placed two OLT, and then through the two light direction using POS connection, so that each uplink of the ONU through the dual PON port for $1+1$ redundancy protection to ensure stable operation of equipment. Communication Interface and Bandwidth Requirement Analysis. The main function of distribution automation communication is to provide communication service for the control switch equipment 
on the distribution network. This is a high demand for the security and stability of the EPON device. The ONU of the optical network unit must adopt the industrial design. The interface is the uplink dual PON port, the downstream Ethernet interface and the RS232 / 485 interface. This shows that the ONU is in the communication interface To meet the requirements of the existing distribution network monitoring terminal (DTU/FTU/TTU).

\section{Conclusion}

At present, the intelligent distribution network is the focus of the development of power grids, and distribution network automation is an important part of intelligent distribution network, to achieve distribution automation needs communication technology support, with electrical communication network as an important part of distribution network automation system, Which is responsible for the connection between all levels of the distribution network, so it is very important to realize the automation of distribution network. This paper analyzes the demand of distribution automation from the aspects of bandwidth, reliability and security. With the construction of communication network, advanced communication technology is needed to realize. There are many communication technologies that can be used in distribution network communication, including optical fiber communication, power line carrier communication, wireless public network communication, wireless private network communication and so on. The research and comparison of these communication technologies summarize the applicable technologies at all levels of the distribution network. And it constructs an EPON-based distribution automation communication scheme. The feasibility analysis is carried out from the aspects of communication medium, network structure, communication interface and bandwidth, service protection capability and construction cost respectively. An EPON system based on AES algorithm is given Communication encryption scheme. Finally, the validity of the EPON topology is analyzed. According to the results of the effectiveness analysis, the EPON topology is suitable for the construction of the distribution network.

\section{Acknowledgements}

Project name: intelligent distribution automation application research

Jiangxi Province Department of Education Science and Technology Program key projects

No.:GJJ151234

\section{References}

[1] Li Tinghua, Huang Ming, Shi Jihong, Chang Jun, Yu Jiang, Wu Wei.We are based on WiMax urban distribution network communication scheme and its application [J]. Power System Communications, 2010 (08)

[2] Sun Zhongwei, Ma Yaining, Wang Yirong, Huosi Tian, Xu Guangnian. EPON-based distribution network automation communication system and its security mechanism [J]. Power System Automation, 2010 (08)

[3] Li Ruide, Ma Yonghong, Wang Yirong. EPON access protection architecture and its cost-effective analysis [J]. Optical Communication Technology, 2010 (03)

[4] Xu Bingyin, Li Tianyou, Xue Yongdian. Intelligent distribution network and distribution automation [J]. Power System Automation, 2009 (17)

[5] Wang Haiyan, Zeng Jiang, Liu Gang. Inspiration of China's automatic distribution network construction model to China's distribution network [J]. Power System Protection and Control, 2009 (11) 